\title{
Bemerkungen zum antiken Homertext
}

\author{
ANTONIOS RENGAKOS
}

In den letzten vier Jahrzehnten hat sich die antike Homerphilologie zweifellos zu einem der am intensivsten erforschten Gegenstände der Klassischen Philologie entwickelt. Textausgaben, Fragmentensammlungen, Spezialuntersuchungen oder Gesamtdarstellungen und nicht zum wenigsten das Lexikon des frügriechischen Epos haben wichtige Resultate erzielen können, aber auch heftige Kontroverse ausgelöst. Trotzdem wird man mit der Behauptung kaum fehlgehen, daß sich in jüngster Zeit in wichtigen Fragen eine communis opinio abzuzeichnen scheint. Das Ziel dieses Beitrags ist die kritische Bestandsaufnahme des Geleisteten, aber auch der Versuch, zu noch ausstehenden Problemen Stellung zu nehmen. Ich werde mich dabei auf zwei Probleme konzentrieren, ein allgemeines, und ein spezielles - beide sind für die Geschichte der Philologie von nicht geringer Bedeutung.

Zuerst das allgemeine Problem: Im Blickpunkt des Forschungsinteresses steht nach wie vor die Kardinalfrage der antiken Homerphilologie, das Problem der Arbeitsweise und der Methode der Alexandriner, konkreter gesagt die Frage, ob die Homerkritiker Handschriften verglichen haben oder ob ihre Lesarten auf Konjektur beruhen; den Anlass zur Behandlung dieser allgemeinen Frage liefert uns ein engstens mit ihr verbundenes spezielles Zetema der antiken Homerkritik, dasjenige der Quellen des Didymos, das jüngst (wieder) kontrovers diskutiert worden ist. Das zweite Spezialproblem, das ich ebenfalls besprechen möchte, ist das Problem der konkreten Gestalt der zenodoteischen Homerausgabe.

1.

Beginnen wir mit der Frage nach der wahren Natur der Lesarten, welche die antiken Zeugen Gelehrten wie Zenodot, Aristophanes von Byzanz oder Aristarch zuschreiben. Mehrheitlich - es genügt die Namen Haslam, Montanari, Nagy, Nickau, Pfeiffer oder Martin Schmidt zu nennen - ist zwar die Forschung von einigen extremen Ansichten abgerückt; ich meine einerseits die frühere, von van der Valk vertretene Theorie, welche die antiken Lesarten 
alexandrinischer Provenienz pauschal als wertlose Konjekturen verwarf, ${ }^{1}$ aber auch die jüngere Theorie van Thiels, nach der die meisten Lesarten der Alexandriner als bloße Hinweise auf Parallelstellen oder Glossen zu interpretieren seien, welche erst Mittelsmänner wie Aristonikos, Didymos usw. als Textvarianten mißverstanden hätten. ${ }^{2}$

Ein wirklicher Konsens ist aber nicht in Sicht: vor einigen Jahren hat nämlich eine gewichtige Stimme, diejenige Martin Wests, eine ebenfalls extreme Theorie aufgestellt, nach welcher einerseits alle zenodoteischen Lesarten urkundlichen Ursprungs seien (ganz einfach weil sie in der ionisch gefärbten Handschrift, welche die Grundlage seines Textes gebildet haben soll, vorhanden waren), andererseits Aristarch für die Konstitution seiner Homerausgabe(n) keine Handschriften verglichen habe. ${ }^{3}$

Mit Wests Ansichten werden wir uns später ausführlicher auseinandersetzen, an dieser Stelle sei jedoch zunächst betont, dass alte und neue Theorien dieser Art von der falschen Voraussetzung ausgehen, dass wir allgemeine Kriterien besitzen, auf Grund deren zuversichtlich zwischen beiden Arten von Lesarten (d.h. zwischen echten [ $=$ durch Handschriften überlieferten] Varianten und Konjekturen) unterschieden werden kann. Wie aber besonders Nickau in seiner Zenodot-Monographie gezeigt hat, ${ }^{4}$ Konjekturen im Homertext als Eigentum eines bestimmten alexandrinischen Kritikers zu erweisen ist unmöglich - man kann nämlich nicht ausschliessen, dass der betreffende Kritiker sie, unwissend über ihren wahren Charakter, in einem älteren Text vorgefunden hat.

Aus diesem Grund ist auch die Faustregel wertlos, die, in verschiedenen Varianten formuliert, besagt, dass die von unserem Vulgatatext abweichenden oder zumindest die durch weitere frühe Zeugnisse nicht unterstützten Lesarten der Alexandriner auf Konjektur beruhen: ${ }^{5}$ erstens ist es nicht möglich, zu jeder alexandrinischen Variante den Nachweis zu erbringen, dass die Variante unse-

1 M. van der Valk, Researches on the Text and Scholia of the Iliad, Leiden 1963.

2 Vgl. H. van Thiel, Zenodot, Aristarch und andere, ZPE 90, 1992, 1-32; Verf., Der Homertext und die hellenistischen Dichter, Stuttgart 1993, 17ff.; M. Schmidt, Variae lectiones oder Parallelstellen: Was notierten Zenodot und Aristarch zu Homer?, ZPE 115, 1997, 1-12; H. van Thiel, Der Homertext in Alexandria, ZPE 115, 1997, 13-36; F. Montanari, Zenodotus, Aristarchus, and the Ekdosis of Homer, in: G. W. Most, Editing Texts - Texte edieren, Göttingen 1998, 4ff.

3 M. L. West, Studies in the Text and Transmission of the Iliad, München-Leipzig 2001; vgl. die Rezensionen von Verf., BMCR 2002.11.15 und G. Nagy, Gnomon 75, 2003, 481-501, sowie Wests Antwort in BMCR 2004.04.17.

4 K. Nickau, Untersuchungen zur textkritischen Methode des Zenodotos von Ephesos, BerlinNew York 1977, 45; 48.

5 R. Janko, The Iliad: A Commentary, vol. IV: Books 13-16, Cambridge 1992, 25: "I agree with van der Valk that most readings where the Alexandrians lack support in the papyri and early codices are conjectures"; vgl. P. Cauer, Grundfragen der Homerkritik, Leipzig 1921-23, 64: "Welche seiner [d.h. Aristarchs] Lesarten sind Konjekturen? Die meisten Chancen haben solche Lesarten, mit denen Aristarch ganz allein steht". 
rer Vulgata voralexandrinisch und somit Zenodot, Aristophanes oder Aristarch auch bekannt gewesen sei, und zweitens können die sogenannten frühen Zeugnisse (z.B. die von den Scholien zitierten antiken Ausgaben oder die vorhandenen frühptolemäischen Papyri) nur mit einer sehr kleinen Zahl alexandrinischer Varianten verglichen werden. Ebenfalls willkürlich ist die Unterscheidung zwischen beiden Arten von Varianten auf Grund ihrer Qualität, d.h. die Annahme, gute Varianten gingen auf die Verwendung älterer Handschriften, schlechte dagegen auf konjekturale Tätigkeit zurück.

Schliesslich, auch Pasquali's Grundsatz ist haltlos, der besagt: "là dove una lezione di Aristarco è contaposta a una del suo maestro Aristofane o più spesso a una di Zenodoto, è estremamente improbabile che tutt'e due siano congetture: al più sarà congettura l'una; anzi quasi sempre, poichè ... gli editori alessandrini erano critici conservatori, non sarà nessuna delle due". ${ }^{6}$ Pasquali's Überzeugung, die Alexandriner seien konservative Kritiker gewesen und deshalb würden beide Lesarten aus zu Rate gezogenen Handschriften stammen, kann natürlich einen stichfesten Beweis nicht ersetzen.

Das Problem läßt sich also nicht auf Grund von diversen Eigenschaften der überlieferten alexandrinischen Varianten pauschal lösen, sondern nur grundsätzlich. Es muss der Beweis geführt werden, daß für die Alexandriner die Verwendung von Handschriften bei der Konstitution ihrer Homerausgaben eine zumindest wahrscheinliche Praxis darstellte. Welche Mittel besitzen wir, um diesen Beweis führen zu können? Es sind dies eine ganze Reihe von Indizien.

Das erste Indiz ist ein Analogieschluss aus den Gepflogenheiten der Schreiber von Papyri. S. West ${ }^{7}$ und Haslam ${ }^{8}$ haben darauf hingewiesen, daß einige frühptolemäische Homer-Papyri Spuren von Kollation mit anderen Homertexten bewahren. Einige Beispiele: im berühmten Ilias-Papyrus 12 aus der Mitte des 3. Jhdts. v.Chr. fügt ein zweiter Schreiber Varianten hinzu; West äußert sogar vorsichtig die Vermutung, daß diese Varianten aus mehreren verglichenen Texten stammen könnten, daß sie eine Art von ,,primitivem apparatus criticus" darstellen. ${ }^{9}$

Dasselbe Bild bieten der Odyssee-Papyrus 31 (2. Hälfte 3. Jhdts. v.Chr.), dessen Schreiber seinen Text mit (nicht besseren) Varianten aus einem anderen Text sowie mit Korrekturen versehen hat, oder der Ilias-Papyrus 51 (1. Jhdt. v. Chr.), zu dem wiederum S. West folgendes bemerkt: ${ }^{10}$ "it looks as if the signs were originally inserted, whether in this papyrus or in an ancestor, by someone

6 G. Pasquali, Storia della tradizione e critica del testo, Firenze 1952, 214.

7 The Ptolemaic Papyri of Homer, Köln-Opladen 1967, 136ff; 223; 263.

8 Homeric Papyri and Transmission of the Text, in: I. Morris-B. Powell, A New Companion to Homer, Leiden 1997, 65 f.

9 S. West, a.a.O. 137.

10 a.a.O. 133. 
who collated his copy with a Vulgate text provided with marginal signs according to the conventional system".

Nur nebenbei sei bemerkt, daß Spuren nachträglicher Korrekturen und Varianten auch in anderen frühptolemäischen, nicht-homerischen Papyri zu finden sind, etwa im Milaneser Posidippus-Papyrus, und daß die Fälle im 2. und 3. Jhdt. n. Chr. sich deutlich vermehren. ${ }^{11}$ Es drängt sich deshalb die Frage auf, die Montanari vor einigen Jahren gestellt hat: ${ }^{12}$ Ist es realistisch anzunehmen, dass eine bei der Herstellung von Kopien geläufige Praxis, die den Vergleich zwischen verschiedenen Vorlagen und die Eintragung von Korrekturen vorsah, den alexandrinischen кpıтıкоí nicht als ein Modell ihrer eigenen Aufgabe erscheinen würde, m.a.W. ist es glaubhaft anzunehmen, dass, wenn der diorthotes eines Scriptoriums den Vergleich von Kopien und deren Korrigierung als Teil seiner Techne betrachtete, diese Methode nicht durch den neuen Typus des diorthotes übernommen wurde, der sich als Gelehrter mit dem Homertext beschäftigte?

Einen zweiten Analogieschluss liefern uns die alexandrinischen DichterGelehrten, insbesondere Apollonios von Rhodos und Kallimachos. Eine stattliche Zahl alexandrinischer Lesarten wurde durch das Zeugnis ihrer Werke, die zahllose Nachahmungen homerischer Ausdrücke, Verse, Motive oder Szenen aufweisen und deshalb eine außerordentlich reiche Sammlung der memoria Homerica darstellen, als urkundlichen Charakters erwiesen. Es werden bezeugt:

- Bindefehler zur älteren homerischen Tradition (die Verse 3.881f. aus den Argonautika des Apollonios sind ein "Zitat" von Od. 6.102ff. in der Version des Peripatetikers Megakleides [4. Jhdt. v. Chr.]),

- die übereinstimmende Verwendung einer homerischen Variante durch einen hellenistischen Dichter, einen frühptolemäischen Papyrus und eine alexandrinische Ausgabe (z.B. ahmt Argon. 4.1571f. Il. 6.4 nach, und zwar in der Version von Pap. Il. 410 [= P. Hibeh 193, ca. 270-230 v.Chr.] und von Pap.

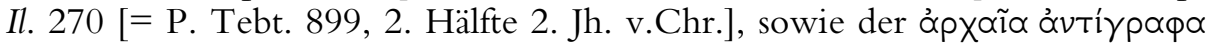
und der ersten Aristarch-Ausgabe),

- das gleichzeitige Vorkommen der Lesart der Vulgata und einer von ihr abweichenden Variante (z.B. Argon. 2.930-45 Il. 2.853-5 in der Version der Vulgata und Strabons),

- die klare Vorwegnahme von Lesarten, die uns bislang unter den Namen späterer Homerkritiker bekannt waren (z.B. Argon. 3.1088f. nach Od. 6.9f. mit Rhianos' Lesart, oder Call. fr. 177.17 Pf. Od. 12.252 mit Kallistratos' Variante). ${ }^{13}$

11 F. Montanari, Ekdosis alessandrina: il libro e il testo, Exemplaria Classica 1, 2009,146ff.

12 Alexandrian Homeric Philology. The Form of the Ekdosis and the Variae Lectiones, in: M. Reichel-A. Rengakos (Hrsg.), Epea pteroenta. Beiträge zur Homerforschung. Festschrift ... W. Kullmann, Stuttgart 2002, 135.

13 Die vier genannten Fälle werden in Verf., Der Homertext..., 128; 154f.; 129; $127 f$. besprochen. 
Wenn also zwei Dichter-Gelehrten der frühptolemäischen Zeit wie Apollonios und Kallimachos für ihre Dichtungen sich nicht mit einer Kopie des Homertextes begnügen, sondern mehrere zu Rate zogen, so dürfen wir wohl annehmen, dass dieselbe Vorgehensweise für die professionellen HomerKritiker selbstverständlich gewesen sein muss.

Ein drittes Mittel, unsere Frage zu lösen, ist der Versuch, die Herkunft alexandrinischer Varianten aus älterer Tradition zu beweisen, m.a.W. echte Bindefehler zur älteren Homerüberlieferung ausfindig zu machen. Seit Wolf, Düntzer und Wecklein gibt es zwar eine Reihe von mehr oder weniger aussagekräftigen, immer wieder genannten Fällen, welche die zenodoteische Ausgabe betreffen, wirklich überzeugend ist jedoch nur eine sehr geringe Anzahl unter ihnen. Es muss gleich hervorgehoben werden, dass dies nicht weiter störend ist, weil es nur auf das Grundsätzliche ankommt: überspitzt formuliert, genügt es nachzuweisen, dass z.B. zwischen den als zenodoteisch überlieferten Lesarten und der älteren Homerüberlieferung ein einziger Bindefehler existiert, um grundsätzlich die Verwendung von Handschriften durch den Ephesier

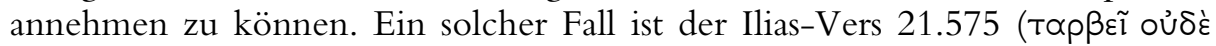

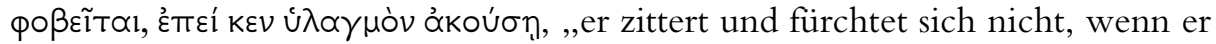
das Gebell hört"), wo unsere handschriftliche (und auch die antike) Vulgata

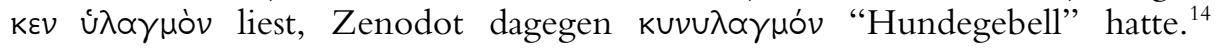

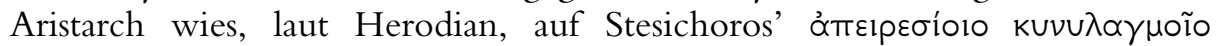
(PMGF 255) hin, d.h. er stellte fest, daß Zenodots Lesart sich respektablen Alters erfreute, und verwarf sie trotzdem aus semasiologischen Gründen.

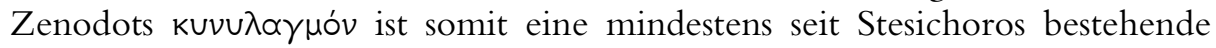
Variante und keine Konjektur des Ephesiers. Auch mit dem vielberufenen

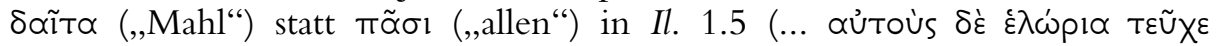

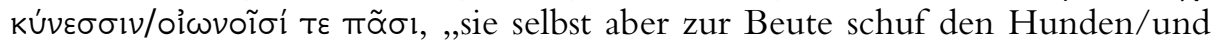
allen Vögeln“ bzw. „den Vögeln zum Mahl“) folgte Zenodot einer Lesart des 5. Jhdts., welche den drei großen Tragikern bekannt gewesen ist (vgl. A. Suppl. 800f., S. Ant. 29-30, E. Ion 504f., Hec. 1078). ${ }^{15}$ Was die Auslassungen von Versen betrifft, so gibt es wiederum mindestens einen sicheren Fall, wo Zenodot älterer Überlieferung gefolgt sein muss. Den Vers Il. 21.195 hat bereits der Peripatetiker Megakleides (4. Jhdt.) nicht berücksichtigt, so daß sich Zenodot, der ihn laut Aristonikos z.St. "nicht schrieb", aller Wahrscheinlichkeit nach auf handschriftlicher Grundlage stützte.

Ausser diesen drei indirekten Indizien gibt es auch ein viertes, diesmal direktes, das die antiken Homerscholien selbst liefern. Sie bezeugen auf unmißverständliche Weise, daß die Alexandriner sowohl Handschriften verglichen als auch Konjekturen vorgenommen haben. Damit sind wir aber bei einem der

14 Nickau, Untersuchungen ... 34f.

15 R. Pfeiffer, Geschichte der Klassischen Philologie. Von den Anfängen bis zum Ende des Hellenismus, München 1978, 142ff.; anders van der Valk, Researches ..., Bd. II, 66ff. und Nickau, Untersuchungen ..., 42, Anm. 32. 
beiden Spezialproblemen gelangt, demjenigen der Quellen des Didymos, das durch das bereits erwähnte Buch von M. West wieder aktuell geworden ist. Es lohnt sich kurz Wests zentrale These in Erinnerung zu rufen, denn, obwohl die Reaktionen auf sie eher negativ ausgefallen sind, ${ }^{16}$ es auch nicht an zustimmenden Urteilen gefehlt hat. ${ }^{17}$

\section{2.}

Wests Theorie über die Quellen des Didymos greift übrigens z.T. eine bereits durch Karl Lehrs vorgetragene Auffassung auf, ${ }^{18}$ der seinerzeit Arthur Ludwich energisch entgegengetreten ist. ${ }^{19}$ West behauptet nun, die Analyse des Wortlauts der didymeischen Scholien führe zu dem Schluss, dass Aristarch außer der zenodoteischen und der aristophanischen (sowie gelegentlich der Lesarten des Philitas, des Apollonios Rhodios und des Eratosthenes) keine weiteren Ausgaben oder Handschriften zu Rate gezogen habe und dass es erst Didymos selbst gewesen sei, der folgende, von ihm zitierte Ausgaben direkt benutzt habe: die Ausgaben des Antimachos, des Rhianos (sei es auf direktem Weg oder über Kallistratos), des Kallistratos (dem er Kenntnis auch der Argolike Ekdosis verdankt), des Aristarch, des Seleukos (dem er Lesarten aus der Kypria, der Kretike und der sogenannten Polystichos verdankt), die Massaliotike, die Chia und vielleicht die Sinopike. Aristarchs ekdoseis und hypomnemata entnimmt Didymos lediglich die Lesarten Zenodots und des Aristophanes. ${ }^{20}$ Aristarch hat also, so die Konsequenz von Wests Theorie, für seine Homerausgabe ausschliesslich innere, Konjektural-, keine äußere, diplomatische Kritik geübt.

Die entscheidende Frage sei sofort gestellt: wird Aristarch in den Didymosoder Aristonikosscholien als andere Ausgaben konsultierend zitiert? Nur nebenbei sei jetzt schon folgendes sich zu fragen erlaubt: wie wahrscheinlich ist es, dass, wie West annimmt, ausgerechnet die drei bedeutendsten Homerkritiker, Zenodot, Aristophanes und Aristarch, keine Handschriften verglichen haben und dass dies als erster eine Nebenfigur der alexandrinischen Homerphilologie wie Kallistratos oder die Kompilatoren der augusteischen Zeit (Didymos) getan haben sollen? Doch zurück zu den Zeugnissen der Scholien.

16 J.-F. Nardelli, Éditer l'Iliade. La transmission et ses débats: perspectives critiques, Gaia 5, 2001, 62f.; R. Janko, Seduta di chiusura, in: F. Montanari (Hrsg.), Omero tremila anni dopo, Roma 2002, 659; G. Nagy, Rez. West, Gnomon a.a.O.; F. Montanari, La filologia omerica antica e la storia del testo omerico, in: A. Bierl-A. Schmitt-A. Willi, Antike Literatur in neuer Deutung (FS Latacz), München-Leipzig 2004, $129 \mathrm{f}$.

17 R. Führer-M. Schmidt, Homerus, Ilias rec. Martin L. West, GGA 253, 2001, 4ff.

18 K. Lehrs, De Aristarchi studiis Homericis, Leipzig 1882, $26 f$.

19 A. Ludwich, Aristarchs homerische Textkritik (nach den Fragmenten des Didymos dargestellt und beurtheilt), Bd. I, Leipzig 1884, 43f.

20 Siehe die zusammenfassende Tabelle West, Studies 84. 
Die Abgrenzung aristarchischer Fragmente in den Homerscholien ist ein notorisches Zetema (nicht von ungefähr besitzen wir immer noch keine Fragmenten-Sammlung Aristarchs!), ich will deshalb zunächst nur die wirklich unumstrittenen Fälle besprechen. Ich lasse deshalb das in Wests sowie Schmidts und Führers Augen zweifelhafte Didymosscholion zu Il. $1.423-424$ beiseite, ${ }^{21}$

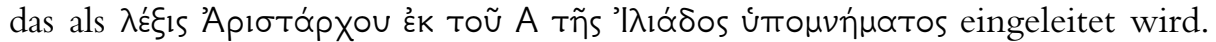

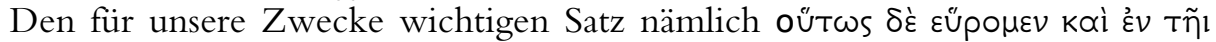

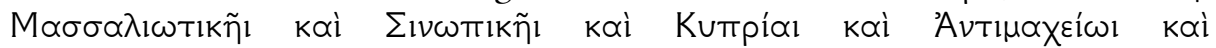

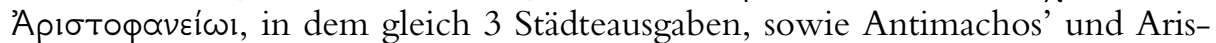
tophanes' Ausgabe zitiert werden, lassen die genannten Forscher nicht als Teil

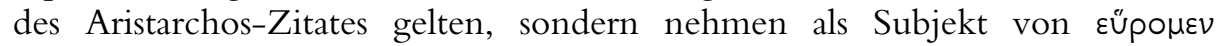
Didymos selbst an - ich finde zu Unrecht, eine detaillierte Diskussion dieses wichtigen Zeugnisses würde aber zu weit führen.

Konzentrieren wir uns auf zwei andere Zeugnisse, in denen auf unmißverständliche Weise davon die Rede ist, daß Aristarch die Wahl seiner Lesart vom Vergleich mehrerer Handschriften abhängig macht:

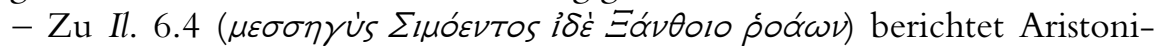

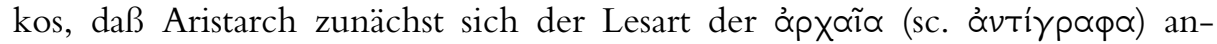

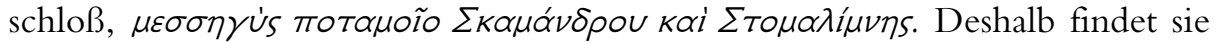

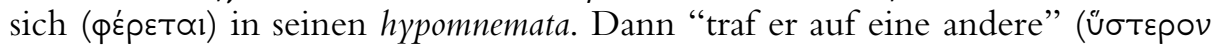

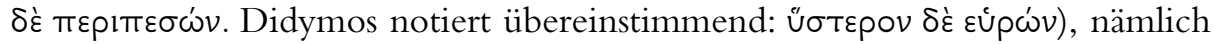

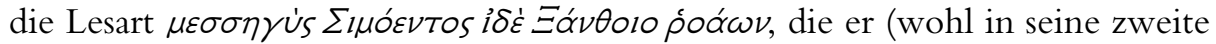
Ausgabe) übernahm ( $\varepsilon \gamma \rho \propto \psi \varepsilon)$.

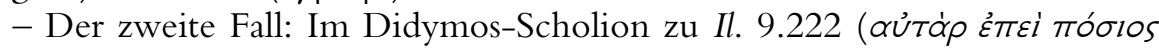

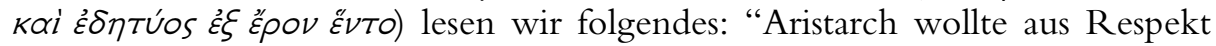
vor der Lesart $\xi^{\xi} \xi \xi \xi \rho \circ \varepsilon^{\prime \prime} \nu T 0$, die er in mehreren Ausgaben vorfand, nichts än-

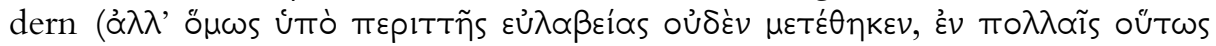

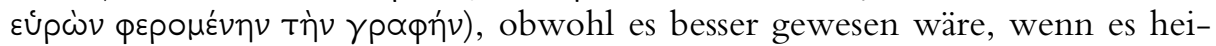

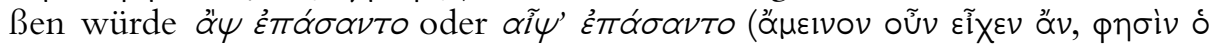

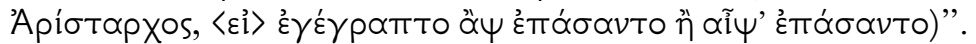

In einer anderen Reihe von Fällen notieren Aristonikos bzw. Didymos, Aristarch berichte ( $(\eta \sigma i)$ ), "einige" Ausgaben (der verwendete Ausdruck ist Tıvย́s oder દ้vıı) würden diese oder jene Lesart bieten; ich zitiere Didymos zu

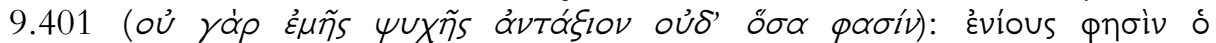

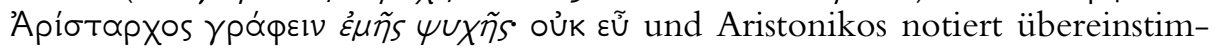

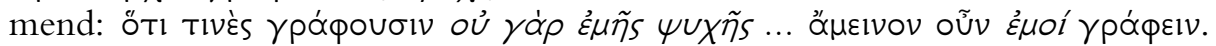
Insgesamt gehören dieser Kategorie fünf Fälle, die Scholien zu den Ilias-Versen 3.230a, 9.159, zum genannten Vers 9.401, zu 11.142 und zu 16.105.

Aus den angeführten Stellen ist nur ein Schluss möglich: nach seinen eigenen Worten berücksichtigt Aristarch bei der Konstitution seines Homertextes Lesarten aus anderen Homerausgaben, die er entweder verwirft oder - was

21 West, Studies 70f.; Führer-Schmidt, GGA 5f. (mit der früheren Literatur). Die Gegenposition: Nagy, Gnomon 490f. 
seltener bezeugt ist - denen er folgt - in einem Fall widerwillig und ausdrücklich nur aus dem Grund, weil die betreffende Lesart von der Mehrheit der ihm zur Verfügung stehenden Handschriften überliefert wurde. Dass die unbestimmten Bezeichnungen tıvés oder हैvıı von Aristarch selbst stammen, wird durch den Zitatcharakter der erwähnten Scholien bewiesen. Wie aber Ludwich gezeigt hat, ${ }^{22}$ ist es bisweilen möglich, durch die Kombination der Didymos- und der Aristonikosscholien eine oder mehrere dieser ungenannten Ausgaben zu identifizieren, was wir hier nicht weiter verfolgen wollen; nur nebenbei sei bemerkt, dass sich unter diesen Ausgaben erwartungsgemäss die

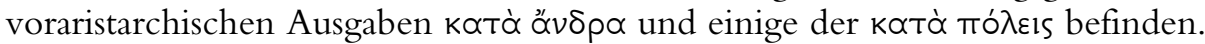
Entgegen Wests Meinung hat also Aristarch ältere Ausgaben - erinnert sei an

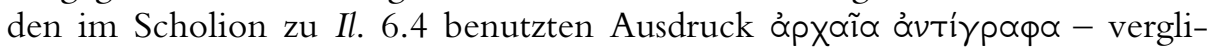
chen.

Bislang haben wir Zeugnisse besprochen, die Aristarch mit der direkten Überlieferung des Homertextes beschäftigt zeigen. Ebenso interessant sind aber diejenigen Scholien, die zeigen, dass die Alexandriner auch die indirekte Überlieferung der Ilias und der Odyssee berücksichtigt haben, d.h. die Homerzitate in der archaischen, der klassischen und besonders der hellenistischen Dichtung. ${ }^{23}$

Einen ersten Fall haben wir bereits kennengelernt: Aristarch weist zum Vers 575 aus Ilias 21 auf Stesichoros hin, dessen Homernachahmung die zenodoteische Lesart bestätigt, die er aber selber (Aristarch) aus semasiologischen Gründen ablehnt.

Einen zweiten Fall stellen die Odyssee-Verse 6.244f. dar (es handelt sich um

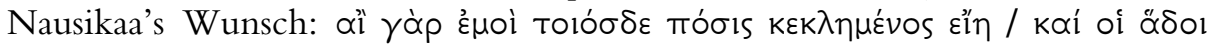
aỦTó $\left.\theta_{1} \mu \dot{i} \mu v \varepsilon ı v\right)$ : laut schol. HQ z.St. hat Aristarch die zwei Verse athetiert, den ersten aber nur zögernd, weil ihn auch Alkman zu benutzen scheint (fr. 81

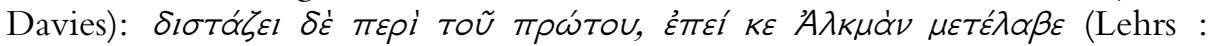

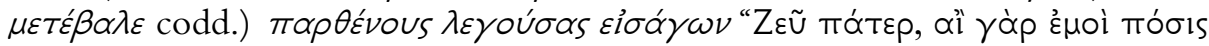
हïn”. Wieder trifft Aristarch eine textkritische Entscheidung unter ausdrücklicher Berücksichtigung der indirekten Überlieferung.

Zwei weitere Dichter zieht Aristarch bei seiner Entscheidung zu Rate, ob

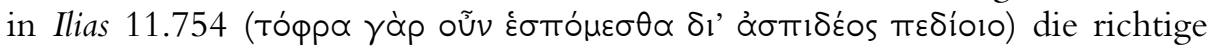
Form des Adjektivs ớ $\sigma \pi \_\delta$ śs oder $\sigma \pi 1 \delta \varepsilon \dot{s}$ ist. In einem wörtlichen Aristarch-

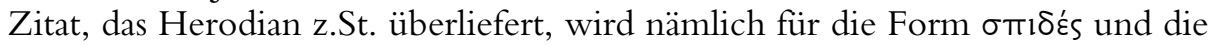

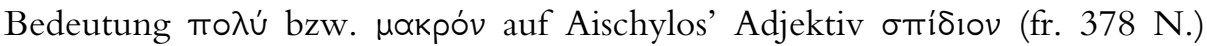

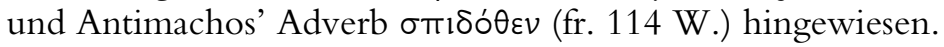

22 Ludwich, Textkritik 2.127f.

23 Weitere Beispiele für die Berücksichtigung von hellenistischen Dichtern durch die alexandrinischen Kritiker bei F. Montanari, Filologi Alessandrini e poeti alessandrini, Aevum Antiquum 8, 1995, 47-63; Verf., Aristarchus and the Hellenistic Poets, Seminari Romani di Cultura Greca 3, 2000, 325-335 und F. Montanari, Callimaco e la filologia, in: Callimaque, Entretiens Hardt 48, Vandoeuvres-Genève 2002, bes. $70 \mathrm{ff}$. 
Das letzte Beispiel betrifft den hellenistischen Epigrammatiker Posidippos

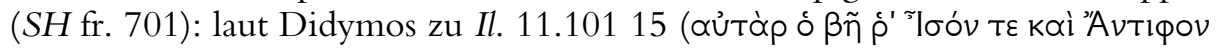
$\dot{\varepsilon} \xi \varepsilon v \propto \rho i \xi \omega v)$ las Zenodot $\beta \tilde{\eta}^{\top}{ }^{\top} \mid \sigma o ́ v$. Aristarch, so Didymos weiter, der der Lesart

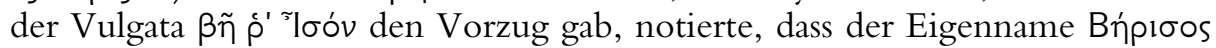
nicht mehr in den Epigrammen des Posidippos überliefert wird, sondern im sogenannten Soros; wahrscheinlich, so fügt Aristarch hinzu, habe Posidippos den Namen eliminiert, nachdem er kritisiert worden ist. M.a.W.: der Epi-

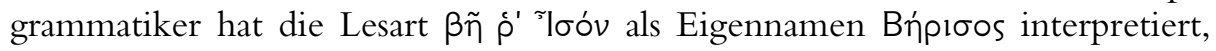
später aber (vielleicht nach der Publikation des zenodoteischen Homertextes Zenodot wird dann derjenige gewesen sein, der Posidippos kritisiert hat) die Lesart verworfen. ${ }^{24}$

Auch Aristarchs Rivale Krates griff auf das Zeugnis der Dichter zurück. In

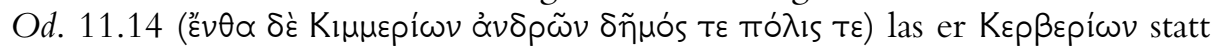
des von der gesamten direkten und indirekten Überlieferung gestützten und

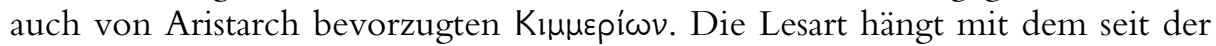
Antike bestehenden Problem der Lokalisation dieses Volkes zusammen. Krates verwarf die Identifizierung mit den historischen Kimmeriern, die ja nicht weit von Griechenland lebten, und lokalisierte sie wahrscheinlich in der Polgegend, wo er auch die Unterwelt wähnte ( K ́⿱㇒㠯 $\beta \varepsilon \rho p o s)$. Eustathios notiert zu Od.

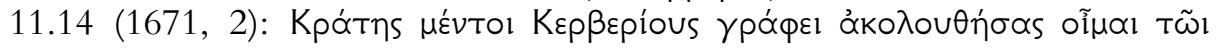

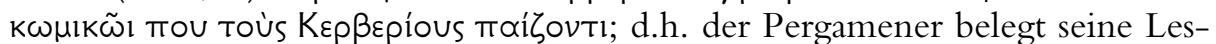
art mit einem Aristophanes- (Ra. 187: in's Kepßepious) und vielleicht auch

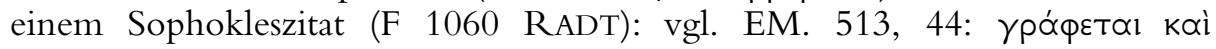

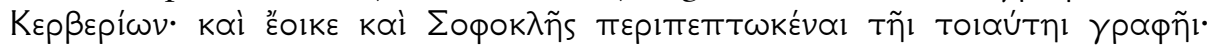

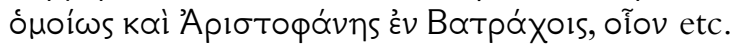

Der Rückgriff der alexandrinischen Philologie auf die direkte und die indirekte Homer-Überlieferung ist also in den Scholien selbt bezeugt. Ausdrückliche Zeugnisse der Konjekturalkritik der Alexandriner sind leider in den Scholien nicht mit Sicherheit zu identifizieren. Es fehlt nämlich eine spezifische

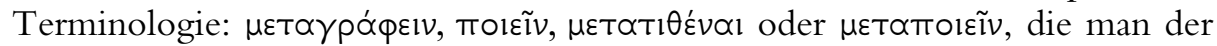
Bedeutung nach als in Frage kommend denken könnte, sind von einfachem

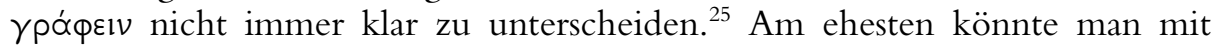
einer gewissen Berechtigung vermuten, dass wir es an Stellen, wo die Wen-

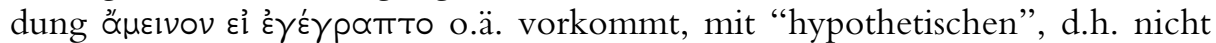
in den Text übernommenen Konjekturen, mit philologischen Gedankenspielen zu tun haben. ${ }^{26}$ Am representativsten ist auch diesmal das Didymos-

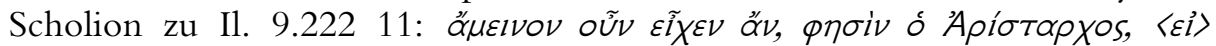

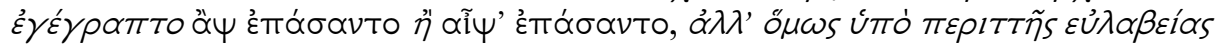

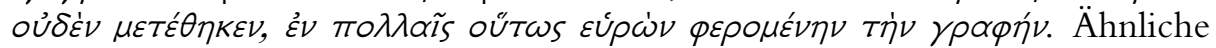

24 Zum Soros vgl. A. Cameron, The Greek Anthology from Meleager to Planudes, Oxford 1993, Appendix V, 369-376; K. Gutzwiller, Poetic Garlands, Berkeley etc., 154ff.

25 Ludwich, Textkritik 2.104f.

26 Pace Ludwich, Textkritik 2.84f. 
Konditionalsätze werden auch in den Didymos-Scholien zu Il. 8.235 oder 16.636 verwendet.

Wir fassen zusammen: nach ausdrücklichem Ausweis Aristarchs - die besprochenen Fälle stammen alle ausnahmsweise aus direkten Aristarch-Zitaten berücksichtigt er bei der Konstitution seines Homertextes sowohl die direkte

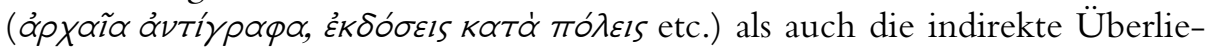
ferung (Homerzitate und -nachahmungen in der archaischen, klassischen und hellenistischen Dichtung). Wie Montanari vor einigen Jahren es formulert hat, handelt es sich bei dieser Vorgehensweise der Alexandriner um einen kulturellen Durchbruch, den Durchbruch von der mechanischen Korrektur eines Textes durch den diorthotes eines Scriptoriums, der Vorlage und Abschrift miteinander verglich, zu der Erkenntnis, dass ein literarischer Text eine Überlieferungsgeschichte hat, während deren er an verschiedenen Stellen entstellt werden könnte, und dass der Originalzustand durch Konjekturen und durch die Auswahl der besseren unter den überlieferten Lesarten wiederhergestellt werden kann.

3.

Kommen wir nun zum zweiten Spezialproblem, das wir untersuchen wollten, der Frage nach der konkreten Gestalt des zenodoteischen Homertextes. Man nahm früher an, ${ }^{27}$ daß es sich um einen fortlaufenden Homertext handelte, der einerseits die zenodoteischen Lesarten enthielt, andererseits an den Rändern Obeloi, das Zeichen für die Athetesen, aufwies; an den Stellen, an denen die Athetesen Zenodots notwendigerweise auch Textveränderungen mit sich zogen, schlug Ludwich vor, daß Zenodot diese Textveränderungen nur supralinear andeutete. Doch beginnend mit Pfeiffer setzte sich eine andere Ansicht durch; Pfeiffer schreibt:28 "Es ist nicht ausgeschlossen, daß Zenodot nach Überprüfung der Handschriften in der Bibliothek einen Homer-Text, der ihm besser als die anderen zu sein schien, als Grundlage auswählte; seine Mängel wird er durch bessere Lesarten in anderen Handschriften oder durch seine eigenen Konjekturen verbessert haben ... Man kann sich kaum ein anderes Vorgehen vorstellen." Und er fährt mit dem Beispiel der italienischen Humanisten fort, die für ihre Ausgabe lateinischer Klassiker einen codex pervetustus zu wählen pflegten, dem sie folgten und den sie gelegentlich durch Vergleich mit anderen Codices sowie durch eigene Konjekturen emendierten. Also: Wahl eines besseren Textes aus den in der Bibliothek zur Verfügung stehenden Handschriften, der durch echte Varianten und Konjekturen emendiert wird -

27 H. Düntzer, De Zenodoti studiis Homericis, Göttingen 1848, 182, Ludwich, Textkritik 2.133f. mit Anm. 113 und G. M. Bolling, The External Evidence for Interpolation in Homer, Oxford 1925, 130.

28 Geschichte 141f. 
Pfeiffer äußert sich leider nicht darüber, wie er sich die konkrete Form vorstellt, in der die Varianten, Athetesen, Auslassungen etc. durch Zenodot in diesen Text eingetragen wurden.

Konkreter sind die Vorstellungen von Nickau:" "dann ist zu fragen, ob Zenodot nicht einen durch Recensio ermittelten Homertext zugrundelegte (der jedoch nicht seinen Vorstellungen von der genuinen Form der Epen entsprach), diesen mit Obeloi versah und $\mathrm{zu}$ ihm Textvorschläge sowie deren Begründung mitteilte". Also wiederum kein autographer Text Zenodots, sondern ein aus den vorhandenen ausgewählter, in dem lediglich die Obeloi eingetragen waren - alle Textvorschläge, Varianten, Auslassungen und Versumstellungen erfolgten mündlich. Nickau führt zwei Gründe an, warum er einen von Zenodot selbst stammenden, fortlaufenden Homertext verwirft, der alle für den Ephesier bezeugten Lesarten enthielt und dem die Obeloi beigegeben waren: zum einen wegen der beiden Stellen (Il. 2.226-228 und 3.334-338a), an denen zenodoteische Athetesen gleichzeitig auch Veränderungen des Kontextes nach sich ziehen, und zum anderen, weil spätere Autoren oft auch über Zenodots Begründüngen seiner Texteingriffe zu berichten in der Lage sind.

Das erste Problem wurde bereits, wie gesagt, durch Ludwich befriedigend gelöst, ${ }^{30}$ der annahm, daß Zenodot solche Texteingriffe "über der betreffenden Zeile durch Überschreiben" andeutete. Zum zweiten Problem läßt sich jetzt schon soviel sagen, daß man zwischen "Begründungen", die natürlich nur mündlich vorgetragen werden konnten, da Zenodot wie bekannt keinen schriftlichen "Kommentar" hinterließ, und Varianten oder Auslassungen unterscheiden muß, die man sich wohl am einfachsten in einem zenodoteischen Homertext eingetragen vorstellen kann. Doch darüber später.

Am gründlichsten hat sich schließlich Franco Montanari mit der Frage der Form der zenodoteischen Ausgabe beschäftigt. In einer Reihe von Arbeiten hat er alle Aspekte des Problems untersucht und ein präzises Bild des zenodoteischen Homertextes entworfen. Für meine weiteren Ausführungen werde ich mich also auf seine kenntnisreiche und besonnene Diskussion stützen.

Auch Montanari nimmt für Zenodots Arbeit einen nicht vom Ephesier selbst stammenden Text, sondern einen mit Sorgfalt ausgewählten, ionisch gefärbten Text als Grundlage (Montanari stimmt hier West zu). Im Gegensatz aber zu Nickau nimmt er - ich glaube zu Recht - an, daß die Texteingriffe schriftlich und nicht mündlich erfolgten: die Athetesen durch die Beigabe des Obelos, die Varianten als Randnotizen und die Versauslassungen durch einen der Mittel, die auch in den literarischen Papyri gängig sind; ich zitiere: "a horizontal or oblique stroke could be drawn above the words or letters to be eliminated, or they could be marked by dots or lines above or below them, or

29 'Zenodotos von Ephesos', RE Xa (1972), 30.

30 Textkritik 2.134; s. auch Düntzer 182 und G. M. Bolling, The Athetized Lines of the Iliad, Baltimore 1944, 73. 
alternatively they could be enclosed within a pair of lines like round parentheses". ${ }^{31}$

Um gleich meine Meinung vorwegzunehmen: ich habe zwei Einwände gegen dieses Bild: einen speziellen, der die Art, mit der Zenodot die Auslassung von Versen markierte, betrifft, und dann einen allgemeineren: warum sollen wir eigentlich nicht an einen unter Zenodots Anleitung gefertigten Text denken, der seine Varianten nicht als Randnotizen, sondern im fortlaufenden Text selbst eingetragen aufwies, und die ausgelassenen Verse nicht als durchgestrichene, unterstrichene oder wie auch immer markierte Verse enthielt, sondern einfach wegließ?

Beginnen wir mit dem Problem der Auslassung von Versen. Die in den Scholien benutzte Terminologie ist wie bekannt schwankend: für diese Art von Texteingriff werden mehrere Ausdrücke verwendet - Nickau hat sie in

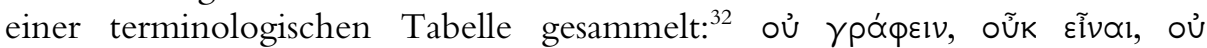

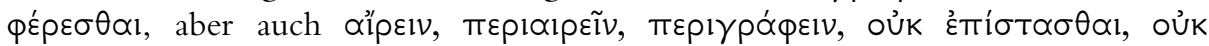

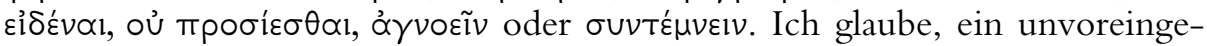
nommener Leser würde gleich zugeben, daß, wie Nickau selbst eingesteht, ${ }^{33}$ diese Ausdrücke den Eindruck vermitteln, "in allen diesen Fällen werde nicht der sozusagen textkritische Apparat Zenodots, sondern sein Homertext beschrieben", m.a.W. sie geben wieder, nicht was aus irgendwelchen Zeichen erschlossen werden könnte, sondern was der Benutzer im zenodoteischen Original oder in dessen Abschriften vorfand bzw. nicht vorfand.

Montanari, der diesen terminologischen Einwand sehr wohl gesehen hat, meint einerseits, dass neben den als auszulassenden markierten Versen ein oủ rpóqsılv oder ähnliches stehen könnte, und andererseits, dass die übrigen Ausdrücke eine vereinfachende und unpräzise Art seien, um diesen Eingriff zu beschreiben. ${ }^{34}$ Ich will zugeben, dass oủ Ypóxpsı gerade noch als Randbemer-

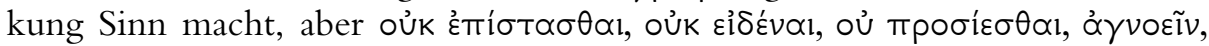

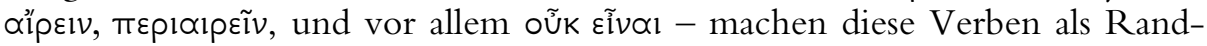
kommentar oder als Beschreibung dessen, was im Text nicht vorhanden ist, mehr Sinn? Sie sind unpräzise und mannigfaltig aus dem einfachen Grund, weil die Auslassung ein "einfacher", direkter und kein raffinierter, indirekter, sozusagen halbherziger Eingriff wie die Athetese ist, und für solch einen Eingriff ist kein spezieller Ausdruck, kein terminus technicus nötig - die Beschreibung des Nicht-Vorhandenseins genügt deshalb vollkommen.

31 Zenodotus, Aristarchus ... 6.

32 Untersuchungen ... 25ff.

33 A.a.O. 13.

34 Zenodotus, Aristarchus ... 7. S. auch F. Montanari, Callimaco e la filologia, in: L. Lehnus-F. Montanari (Hrsg.), Callimaque. Entretiens sur l'antiquité classique 48, Vandoeuvres-Genève 2002, 62ff.. 
Gegen diese wie mir scheint einfachste Lösung könnte man mit Montanari

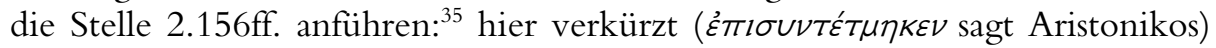

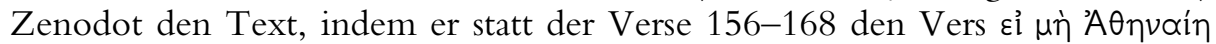

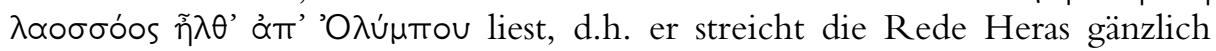

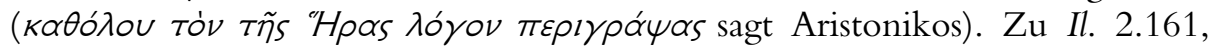
einem also von Zenodot ausgelassenen Vers, wird eine Lesart des Ephesiers

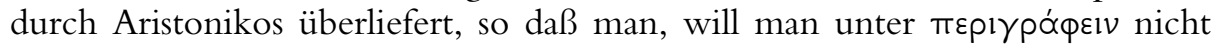
einen speziellen Texteingriff, d.h. den Einsatz von Klammern, verstehen, sich die ausgelassenen Verse als doch im Text vorhanden denken müßte, womit unsere Hypothese von einem Text, in dem die ausgelassenen Verse auch wirklich nicht vorhanden waren, hinfällig würde.

Doch Nickau hat treffend darauf hingewiesen, ${ }^{36}$ daß sowohl hier als auch im zweiten, ähnlich gelegenen Fall der von Zenodot ausgelassenen Verse Il. 2.111-118, wo ebenfalls eine zenodoteische Variante zu einem dieser Verse (111) überliefert wird, es sich um Wiederholungsverse handelt $(2.161=2.177$ und $2.111=9.18$ ) und somit wahrscheinlich sei, dass Aristonikos' Erwähnung der Varianten sich auf das spätere Vorkommen dieser Verse beziehen könnte.

Nichts, so meine Schlußfolgerung, spricht also gegen die Annahme, daß Zenodot die auszulassenden Verse in seinem Text nicht irgendwie graphisch markierte, sondern einfach nicht schrieb. Man bedenke zusätzlich, daß, während Zenodot den Obelos für seine Athetesen einführte, für das Weglassen jedoch von Versen, das gewiß eine viel wichtigere Textentscheidung darstellt, kein kritisches Zeichen überliefert ist. Ließ Zenodot diese Verse in seinem Exemplar einfach stehen und verdammte sie nur mündlich? Und ist es dann wahrscheinlich, daß er ein Zeichen nur für die doch "harmloseren" Athetesen erfunden hätte?

Wenn also die von Zenodot ausgelassenen Verse in seinem Text einfach fehlten, warum sollten seine Varianten als Randnotizen erscheinen und nicht vielmehr im fortlaufenden Text eingetragen sein? Einen Grund dafür kann ich nicht finden. Ich fasse also meine Meinung über das zenodoteische Original zusammen: ein fortlaufender Text, der Obeloi und Varianten enthielt und die eliminierten Verse wirklich ausließ. ${ }^{37}$ Die überlieferten Begründungen dieser Texteeingriffe, darin ist Montanari zuzustimmen, stammen wahrscheinlich aus der mündlichen Tradition seiner Vorträge im Museion. Die bereits zu Aristarchs Zeiten auftretenden Unsicherheiten über einige seiner Varianten sind, ebenfalls mit Montanari, auf die Verwendung von Abschriften der Ausgabe Zenodots zurückzuführen, die wie jede Kopie zuweilen voneinander abwichen.

35 Callimaco 62f. Anm. 10.

36 Untersuchungen 13.

37 M. Haslam, Homeric Papyri and Transmission of the Text, in: I. Morris-B. Powell (Hrsg.), A New Companion to Homer, Leiden 1997, 73. 
Wie anfangs gesagt, ist die alexandrinische Homerphilologie heute dank einer Reihe kommentierter Fragmentsammlungen und spezieller Abhandlungen besser denn je erschlossen. Kontroverse gibt es immer noch, doch alte Probleme dürfen nunmehr als endgültig gelöst gelten - ich erinnere nur an Montanari's elegante Lösung des Zetemas der zwei aristarchischen HomerAusgaben. Das wichtigste ist aber, dass, indem wir in der Lage sind, uns ein genaueres Bild von der alexandrinischen Philologie zu machen und somit sie auch besser zu würdigen, die Homerkritiker nicht mehr subsidiär, als meistens unzuverlässliche Zeugen der Geschichte des Homertextes, sondern um ihrer selbst willen als wichtige Zeugen des kulturellen und intellektuellen Milieus, in dem sie wirkten, erforscht werden. 